Helmholtz resonance in a piezoelectric-hydraulic pump-based hybrid actuator

This article has been downloaded from IOPscience. Please scroll down to see the full text article.

2011 Smart Mater. Struct. 20015010

(http://iopscience.iop.org/0964-1726/20/1/015010)

View the table of contents for this issue, or go to the journal homepage for more

Download details:

IP Address: 141.211.173.82

The article was downloaded on 06/04/2012 at 16:59

Please note that terms and conditions apply. 


\title{
Helmholtz resonance in a piezoelectric-hydraulic pump-based hybrid actuator
}

\author{
Gi-Woo Kim and K W Wang \\ Department of Mechanical Engineering, University of Michigan, Ann Arbor, MI 48109, USA \\ E-mail: gwkim@umich.edu
}

Received 21 June 2010, in final form 3 November 2010

Published 9 December 2010

Online at stacks.iop.org/SMS/20/015010

\begin{abstract}
This paper demonstrates that a hydraulically acting Helmholtz resonator can exist in a piezoelectric-hydraulic pump (PHP) based hybrid actuator, which in turn affects the volumetric efficiency of the PHP. The simulation and experimental results illustrate the effect of Helmholtz resonance on the flow rate performance of the PHP. The study also shows how to shift the Helmholtz resonant frequency to a higher value through changing parameters such as the cylinder diameter and the effective bulk modulus of the working fluid, which will improve the volumetric efficiency and broaden the operating frequency range of the PHP actuator.

(Some figures in this article are in colour only in the electronic version)
\end{abstract}

\section{Nomenclature}

$A \quad$ ram (effective) area $\left(\mathrm{m}^{2}\right)$

$C_{\mathrm{p}} \quad$ capacitance of PZT stack (F)

$C_{\mathrm{m}} \quad$ compliance of PZT stack $\left(=k_{\mathrm{m}}^{-1}\right)$

$C_{1} \quad$ compliance of Helmholtz resonator $\left(=k_{1}^{-1}\right)$

$C_{2} \quad$ compliance of pumping chamber $\left(=k_{2}^{-1}\right)$

$d_{1}$ diameter of double-acting hydraulic cylinder (m)

$d_{2} \quad$ diameter of pumping chamber (m)

$d_{33}$ electro-mechanical charge (coupling) coefficient

$h_{1} \quad$ height of double-acting hydraulic cylinder (m)

$h_{2}$ height of pumping chamber (m)

$i \quad$ electric current (A)

$f \quad$ frequency $(\mathrm{Hz})$

$k_{\mathrm{m}} \quad$ stiffness of PZT stack transducer $\left(\mathrm{N} \mathrm{m}^{-1}\right)$

$k_{1} \quad$ stiffness of Helmholtz resonator $\left(\mathrm{N} \mathrm{m}^{-1}\right)$

$k_{2} \quad$ stiffness of pumping chamber $\left(\mathrm{N} \mathrm{m}^{-1}\right)$

$M_{2}$ effective mass of stiffener with diaphragm $(\mathrm{kg})$

$M_{1} \quad$ effective mass of fluid $(\mathrm{kg})$

$N$ electro-mechanical conversion constant

$n$ number of PZT stack layer

$Q_{1} \quad$ flow rate $\left(\mathrm{cm}^{3} \mathrm{~s}^{-1}\right)$

$s \quad$ Laplace operator

$t \quad$ thickness of PZT stack layer (m)

$V_{o} \quad$ control volume of fluid cavity $\left(\mathrm{m}^{3}\right)$
$V_{\text {AC }} \quad$ AC input voltage (Volt)

$x, \dot{x} \quad$ displacement and velocity $\left(\mathrm{m}, \mathrm{m} \mathrm{s}^{-1}\right)$

$Z_{\mathrm{e}} \quad$ electrical impedance

$Z_{\mathrm{m}} \quad$ mechanical impedance

$Z_{\mathrm{i}} \quad$ complex impedance of subscript

$\beta_{\mathrm{e}} \quad$ effective bulk modulus of working fluid ( $\left.\mathrm{Pa}\right)$

$\mu \quad$ volumetric efficiency

\section{Introduction}

Hydraulic actuation systems play an important role in many industrial applications. However, conventional electrohydraulic actuation systems have many shortcomings and technical limitations that restrict performance. To overcome these difficulties, new devices have been investigated to replace the conventional actuation systems. One of the potential alternatives is to utilize a piezoelectric-hydraulic pump (PHP) concept. The key idea of the PHP-based hybrid actuator (or PHP actuator) is to combine the advantages of piezoelectric transducers (high power density and large load authority) and hydraulic systems (large stroke). Over the past years, several prototype PHP-based actuators have been introduced. Konishi et al [1] have developed a macro-scale PHP using a piezoelectric stack actuator, and recognized that using a flexible diaphragm in the pumping chamber could increase its bandwidth. Different versions of new actuation systems 


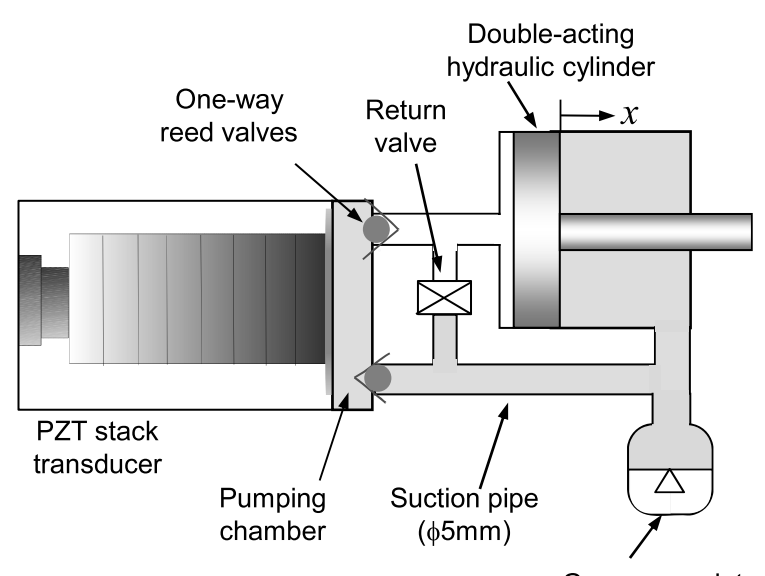

Gas accumulator

Figure 1. Schematic hydraulic circuit of the PHP actuator (gray area: suction flow side).

consisting of a PHP were designed [2-4]. Sirohi et al [5] used low voltage PZT stacks at higher frequencies to more effectively utilize their high power density. In addition, they substituted the ball-type check valves with reed valves to extend the bandwidth of the one-way valves. To overcome the limitations of passive one-way valves, active check valves have been investigated by several researchers [6, 7]. They developed a PHP that consists of a piezoelectric stack actuator and two piezoelectric unimorph disc valves as one-way valves. Building on these previous efforts, researchers have recently investigated the feasibility of using PHP actuators in various industrial applications; one example is to manufacture a PHP for advanced automotive powertrain control systems [8]. The primary challenge here is to develop a high-performance PHP actuator that can satisfy specific requirements, especially flow rate. Most previous research efforts to improve the flow rate of PHP actuators have focused on increasing the driving frequency up to a few $\mathrm{kHz}$, based on the assumption that the flow rate of the PHP actuator will be proportional to the driving frequency [5]. However, the flow rate of a PHP actuator fluctuates in the high frequency range. The most likely reasons for flow rate fluctuation may be due to the bandwidth limitation of one-way valves and unknown resonances in the system. Moreover, the demand for a high operating frequency requires a high current capacity driver to compensate for the large capacitive load (10-50 $\mu \mathrm{F})$ of the PZT stack transducers. This typically restricts the development of efficient and compact systems, which is required in many industrial applications. Therefore, it is preferable to develop a high-performance PHP actuator that can operate effectively and efficiently in the relatively lower frequency range (i.e. a few hundred $\mathrm{Hz}$ ).

\section{Problem statement and objective}

A schematic diagram of the testbed PHP actuator used in this research is illustrated in figure 1 . To have a compact design, this hybrid actuator needs to be a stand-alone device, featuring a self-constrained hardware configuration. A hydraulic transmission line (i.e. suction pipe) is connected to the

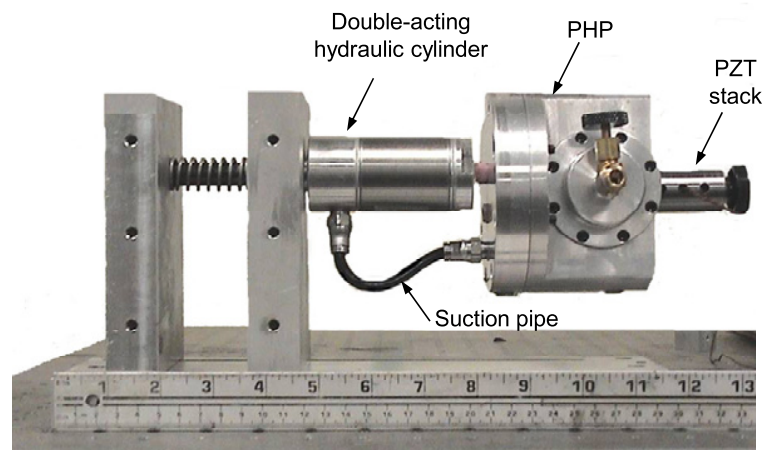

Figure 2. Configuration of the stand-alone PHP actuator.

chambers on both sides of the double-acting hydraulic cylinder to cancel the initial force caused by the gas accumulator pressure. The rigid pipe with a diameter of $0.005 \mathrm{~m}$ is designed to match the outlet impedance (i.e. the outlet port size is also $\varnothing 0.005 \mathrm{~m}$ ). This hardware configuration makes the head side of the double-acting hydraulic cylinder act as an oil reservoir, as opposed to conventional oil tanks. The compactness requirement also rules out the option of using standard commercial accumulators. Instead, in this research a miniature gas accumulator is incorporated by using a rubber diaphragm. Based on this hydraulic circuit, the prototype PHP actuator is designed and fabricated, as shown in figure 2. As illustrated, this stand-alone prototype allows the elimination of fluid line dynamics and minimizes the flow resistance and opportunity of air inflow.

An approximation method using a hydraulic cylinder attached to the PHP actuator is often utilized to evaluate the flow rate performance of the PHP actuator. The assumption is that the pressure-rise rate of the hydraulic cylinder becomes nearly zero during the fast stroke without loading:

$$
\dot{P}=\frac{\beta_{\mathrm{e}}}{\left(V_{o}+A_{1} x\right)}\left(Q_{1}-A_{1} \dot{x}\right) \approx 0
$$

where $\beta_{\mathrm{e}}$ is the effective bulk modulus, $V_{o}$ is the control volume, $A_{1}$ is the effective (ram) area of cylinder, $x$ and $\dot{x}$ are the displacement (i.e. stroke) and average velocity of cylinder, respectively; $Q_{1}$ represents the flow rate. From equation (1), the no-load flow rate can be estimated as follows:

$$
Q_{1}=A_{1} \dot{x}
$$

Since the PHP is a positive displacement type pump that utilizes a combination of the reciprocating action of a flexible diaphragm and one-way check valves, this pump enables the displacement of fluid by trapping a small fixed amount of fluid in the discharging side. Accordingly, a theoretical flow rate (frequently referred to as the displacement of the pump) can be defined as follows:

$$
Q_{\mathrm{TH}}=\delta \frac{\pi D^{2}}{4} f
$$

where $\delta$ is the effective stroke, $D$ is the diameter of the pumping chamber, and $f$ is the driving frequency. Once 


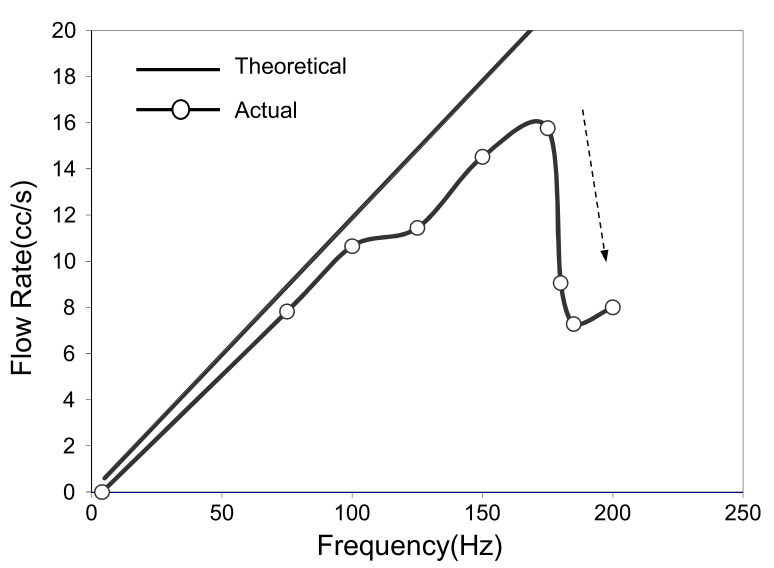

Figure 3. Typical flow rate performance of the PHP actuator $(0.7 \mathrm{MPa}$ accumulator pressure, $120 \mathrm{~V})$.

the theoretical flow rate is defined, the volumetric efficiency becomes one of the key indices for evaluating the flow rate performance of positive displacement type pumps:

$$
\eta=\frac{Q_{1}}{Q_{\mathrm{TH}}} .
$$

To explain the volumetric efficiency, the typical flow rate performance of the prototype PHP actuator is illustrated in figure 3, showing the two flow rates defined in equations (2) and (3). It is obvious that the differences between the theoretical flow rate and the actual measured flow rate are gradually increasing as frequency increases from 0 to $170 \mathrm{~Hz}$. It is well recognized that this phenomenon is common in displacement type pumps. In general, the primary factors that negatively affect the volumetric efficiency of the pump include leakage into the back flow direction and external loads. The volumetric efficiency is also affected by the suction (i.e. inlet) flow dominated by the pipe size and pipe dynamics. At high frequency, the small pipe size of the suction may cause a flow restriction and deteriorated volumetric efficiency. In addition, the different pipe size between suction and discharge may lead to power transmission loss, known as impedance mismatching. As shown in figure 3, the measured actual flow rate suddenly drops when the frequency is beyond a certain range (in this case, beyond $170 \mathrm{~Hz}$ ), which leads to a significant degrading of volumetric efficiency. This undesirable phenomenon has been reported in many other studies on PHP actuators using similar hydraulic circuits [5, 8-10]. Unfortunately, the aforementioned factors do not explain this significant drop in volumetric efficiency. In order to facilitate driving the PHP in the low frequency range $(1-300 \mathrm{~Hz})$, this unwanted phenomenon has to be first examined and the volumetric efficiency needs to be enhanced.

The objectives of this research are (a) to identify the main reason for the degradation of the volumetric efficiency of the PHP actuator in the specific frequency range (e.g. $>170 \mathrm{~Hz}$ for the case shown in figure 3) and (b) to develop methods to improve the PHP's performance. A potential cause for this degradation phenomenon is hypothesized to be the Helmholtz resonance effect, due to the fact that the cavity size of the

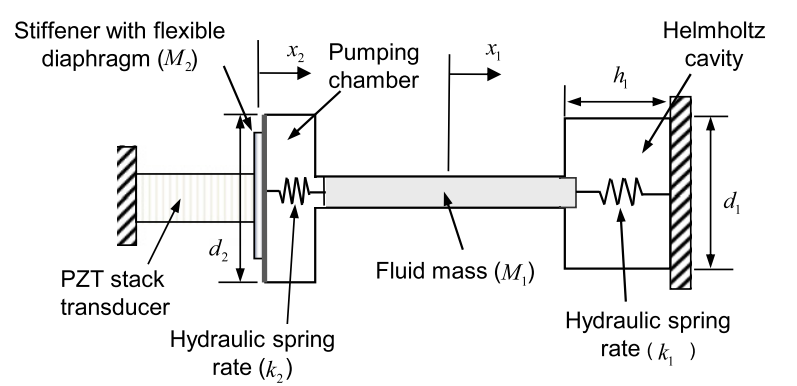

Figure 4. Lumped model for the simplified suction flow rate side.

PHP cylinder is relatively large as compared to the pipe size (a typical Helmholtz resonator configuration). In this study, we verify such a hypothesis by analyzing a Helmholtz resonance model and comparing the results with test data. An equivalent electric circuit model to describe the Helmholtz resonance characteristics is first synthesized. Simulation results are generated by utilizing the derived model and comparing to experimental outcomes. We then identify means to shift the Helmholtz resonance to a higher frequency range through parameter tuning of the model, which leads to the improvement of volumetric efficiency by expanding the effective operational frequency bandwidth.

\section{Helmholtz resonance model}

A Helmholtz resonator, generally known as a large cavity of fluid with an open port, is widely used in a variety of applications to reduce unwanted sound or improve transmission efficiency [11, 12, 14]. For instance, in order to achieve large mass flow during the suction stroke, the Helmholtz resonator is widely used in current internal combustion engines. In a similar manner, the characteristic of suction flow has a great effect on the flow rate performance of the positive displacement type pump since the discharge flow rate depends on the capability to circulate the working fluid, as mentioned in section 2. To explain the Helmholtz resonator, only the suction flow side is considered and simplified by using a lumped model, as shown in figure 4. As noted in section 2, since the cavity size of the cylinder is relatively large compared to the pipe size, it can very likely be viewed as the source of the Helmholtz resonator.

In this lumped model, the dynamics of the reed valves is excluded based on the fact that the first natural frequency of the cantilever type reed valve immersed in fluid is estimated to be much higher than the operational frequency range of the PHP actuator (below $250 \mathrm{~Hz}$ ). Moreover, the motion of the reeds is generally limited by the stopper to prevent excessive deformation, which implies that the orifice in reed valves is fully opening and will not affect the flow rate when the PZT stack transducer is operating. The stiffness of the flexible diaphragm (e.g. spring steel with a thickness of $0.1 \mathrm{~mm}$ ) is also assumed to be very small and negligible since the effective stroke is mainly governed by the PZT stack transducer. From this lumped model, the large chamber on the low pressure (head) side of the double-acting hydraulic cylinder can act as a 
Helmholtz resonator consisting of an effective hydraulic spring rate $\left(k_{1}\right)$ and a fluid mass $\left(M_{1}\right)$. It has been recognized that the fluid effective bulk modulus is a critical parameter that will affect the apparent 'stiffness' of the working fluid [13, 15]. The term stiffness is frequently referred to as the effective hydraulic spring rate in a hydraulic control (or fluid power) system

$$
k_{\mathrm{e}}=\frac{\beta_{\mathrm{e}} A^{2}}{V_{o}}
$$

where $\beta_{\mathrm{e}}$ is the effective bulk modulus, $A$ represents the ram area, and $V_{o}$ is the control volume of the fluid cavity. Note that the inverse of the effective hydraulic spring rate (hydraulic compliance) is different from the hydraulic compliance in the rate of pressure increase (i.e. continuity) equation [15]. From equation (5) and the definition of control volume $\left(V_{o}=A h\right)$, the effective hydraulic spring rates of the PHP actuator shown in figure 4 can be written as follows:

$$
\begin{aligned}
& k_{1}=\frac{\beta_{\mathrm{e}} A_{1}}{h_{1}}=\beta_{\mathrm{e}} \frac{\pi d_{1}^{2}}{4 h_{1}} \\
& k_{2}=\frac{\beta_{\mathrm{e}} A_{2}}{h_{2}}=\beta_{\mathrm{e}} \frac{\pi d_{2}^{2}}{4 h_{2}}
\end{aligned}
$$

where $d_{i}$ and $h_{i}, i=1,2$ are the diameter and height of the two fluid cavities, respectively. In fact, the height of the Helmholtz cavity $\left(h_{1}\right)$ is a time-varying parameter due to stroking. In this analysis, the stroke $(x)$ is set to be small (e.g. $0.005 \mathrm{~m}$ ) to minimize its effect, and is assumed to be quasi-static. From equations (6) and (7), it is evident that the hydraulic spring rate is a function of the effective bulk modulus and geometric variables (such as the diameter of fluid cavity), which can be adjustable parameters for tuning the resonance frequency.

In order to effectively model the coupled electromechanical structure depicted in figure 4, the equivalent electric circuit model is synthesized in figure 5. A co-fired PZT stack driven by an AC voltage is used as an energy transducer in this study. The multi-layered PZT stack transducers are typically made of thin layers (typical $100 \mu \mathrm{m}$ ). They can then be driven by low voltage (i.e. below $150 \mathrm{~V}$ ), compared to standard piezoelectric material-based actuators. In general, $C_{\mathrm{p}}$ represents the electrical capacitance of the PZT stack transducer $(30 \mu \mathrm{F})$. Since the desired normal operational frequency range is far below the resonance frequency of the PZT stack transducer (i.e. $250 \mathrm{~Hz} \ll 40 \mathrm{kHz}$ ), a non-resonant circuit model is utilized in this research for the piezoelectric material. $C_{\mathrm{m}}$ is the mechanical compliance (i.e. the inverse of stiffness $k_{\mathrm{m}}$ ) of the PZT stack transducer. $M_{2}$ and $C_{2}$ represent the effective mass of the stiffener and the fluid compliance (i.e. the inverse of the hydraulic spring rate $k_{2}$ ) of the pumping chamber, respectively. On the other hand, $C_{1}, R_{1}$ and $M_{1}$ are the mechanical properties of the Helmholtz resonator. For instance, $C_{1}$ represents the compliance (i.e. the inverse of the hydraulic spring rate $k_{1}$ ) of the Helmholtz resonator. The fluid mass is estimated by using an approximated pipe volume and fluid density.

An AC voltage is applied to the PZT stack transducer to create the effective force that can stroke the diaphragm in

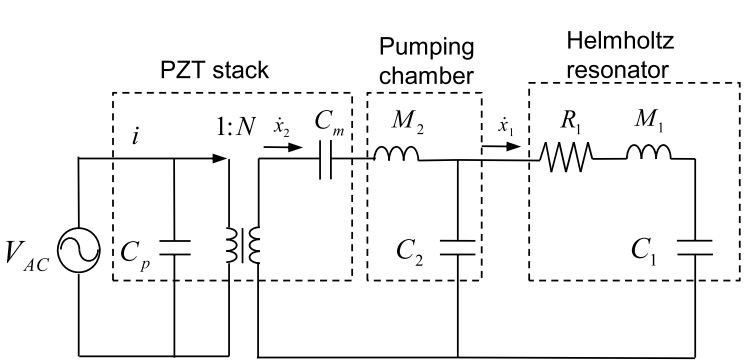

Figure 5. Equivalent circuit model representation.

a pumping chamber. The following ideal transformer will account for this energy conversion:

$$
\begin{gathered}
i=N Q=N A \dot{x} \\
F=N V_{\mathrm{AC}}
\end{gathered}
$$

where $i$ the electrical current, $N$ is the electro-mechanical conversion constant, and $\dot{x}$ is the velocity. The electromechanical conversion constant $N$ can be derived from the constitutive equations of linear piezoelectricity for PZT materials

$$
S_{i j}=s_{i j k l}^{E} T_{k l}+d_{k i j} E_{k} .
$$

In the uniaxial directional case, equation (10) can be reduced to

$$
S_{33}=s_{33}^{E} T_{3}+d_{33} E_{3}
$$

where $S_{33}$ is the mechanical strain, $T_{3}$ is the mechanical stress, $s_{33}^{E}$ is the mechanical compliance of the PZT material, $E_{3}$ is the applied electric field, and $d_{33}$ represents the electro-mechanical charge (coupling) coefficient. For a multi-layered PZT stack transducer with $n$ layers, equation (11) can be rewritten as

$$
\frac{x}{n t}=s_{33}^{E} \frac{F_{\mathrm{ext}}}{A_{\mathrm{p}}}+d_{33} \frac{V_{\mathrm{AC}}}{t}
$$

where $n$ is the number of stack layers, $t$ is the thickness of each layer, $F_{\text {ext }}$ represents the external force, and $A_{\mathrm{p}}$ is the crosssectional area of the PZT stack transducer. Then, equation (12) can be rearranged as follows:

$$
F_{\mathrm{ext}}=k_{\mathrm{m}} x-k_{\mathrm{m}} n d_{33} V_{\mathrm{AC}}
$$

where $k_{\mathrm{m}}$ is the stack stiffness $\left(=A_{\mathrm{p}} / n t s_{33}^{E}\right)$. The second term in equation (13) is called the piezoelectric induced block force of the stack and the electro-mechanical conversion constant $N$ can be defined by

$$
N=k_{\mathrm{m}} n d_{33} .
$$

Then, the electrical impedance of the model can also be converted into the mechanical impedance from equations (8) and (9)

$$
Z_{\mathrm{e}}=\frac{V_{\mathrm{AC}}}{i}=\frac{F / N}{N Q}=\frac{1}{A N^{2}} \frac{F}{\dot{x}} .
$$

In deriving the transfer function for the equivalent circuit model, it is convenient to derive the Laplace-transformed equations directly with the assumption of zero initial conditions. Based on equation (15), the transformer can be 


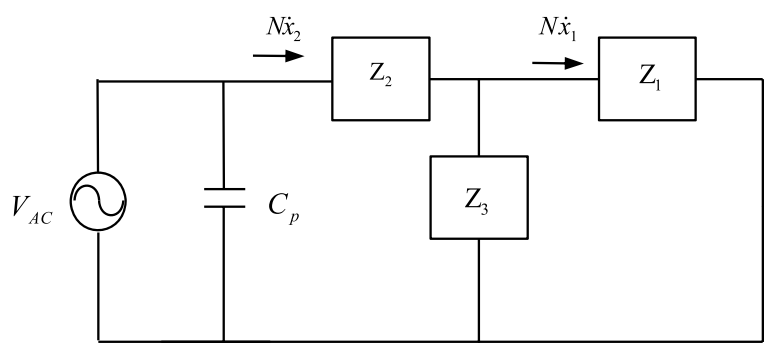

Figure 6. Alternative impedance model representation.

ruled out by using each converted complex impedance part, as shown in figure 6 .

$$
\begin{gathered}
Z_{1}(s)=\frac{1}{A N^{2}}\left(R_{1}+s M_{1}+\frac{1}{s C_{1}}\right) \\
Z_{2}(s)=\frac{1}{A N^{2}}\left(s M_{2}+\frac{1}{s C_{\mathrm{m}}}\right) \\
Z_{3}(s)=\frac{1}{A N^{2}}\left(\frac{1}{s C_{2}}\right)
\end{gathered}
$$

where $s$ is the Laplace operator.

The overall complex impedance $Z_{\mathrm{e}}$ in the Laplace domain can then be obtained using the standard impedance block manipulation method

$$
Z_{\mathrm{e}}(s)=Z_{1}+\left(\frac{Z_{2} Z_{3}}{Z_{2}+Z_{3}}\right) .
$$

Substituting equations (17)-(19) into equation (20) yields

$$
Z_{\mathrm{e}}(s)=\frac{a_{4} s^{4}+a_{3} s^{3}+a_{2} s^{2}+a_{1} s+a_{0}}{A N^{2} b_{1} s}
$$

where

$$
\begin{gathered}
a_{4}=C_{1} C_{\mathrm{m}} C_{2} M_{\mathrm{m}} M_{1} \quad a_{3}=C_{1} C_{\mathrm{m}} C_{2} M_{\mathrm{m}} R_{1} \\
a_{2}=C_{1} C_{\mathrm{m}} M_{1}+C_{1} C_{2} M_{1}+C_{1} C_{\mathrm{m}} M_{\mathrm{m}}+C_{\mathrm{m}} C_{2} M_{\mathrm{m}} \\
a_{1}=C_{1} C_{\mathrm{m}} R_{1}+C_{1} C_{2} R_{1} \quad a_{0}=C_{1}+C_{\mathrm{m}}+C_{2} \\
b_{1}=C_{1} C_{\mathrm{m}} .
\end{gathered}
$$

To evaluate the flow rate output defined in equation (2), the transfer function between the flow rate and the voltage input in Laplace domain is then derived:

$$
\frac{Q_{1}(s)}{V_{\mathrm{AC}}(s)}=\frac{1}{N Z_{\mathrm{e}}} .
$$

The transfer function defined in equation (21) can eventually be rewritten by

$$
\frac{Q_{1}(s)}{V_{\mathrm{AC}}(s)}=\left(\frac{A N b_{1} s}{a_{4} s^{4}+a_{3} s^{3}+a_{2} s^{2}+a_{1} s+a_{0}}\right) .
$$

\begin{tabular}{|c|c|c|}
\hline Symbol & Items (SI unit) & Nominal value \\
\hline$d_{1}$ & $\begin{array}{l}\text { Diameter of double-acting hydraulic } \\
\text { cylinder }(\mathrm{m})\end{array}$ & $0.038,0.05$ \\
\hline$d_{2}$ & Diameter of pumping chamber $(\mathrm{m})$ & 0.03 \\
\hline$h_{1}$ & $\begin{array}{l}\text { Height of double-acting hydraulic } \\
\text { cylinder }(\mathrm{m})\end{array}$ & $0.006,0.01$ \\
\hline$h_{2}$ & Height of pumping chamber $(\mathrm{m})$ & 0.003 \\
\hline$C_{\mathrm{p}}$ & Capacitance of PZT stack (F) & $30 \times 10^{-6}$ \\
\hline$k_{\mathrm{m}}$ & PZT stack stiffness $\left(\mathrm{N} \mathrm{m}^{-1}\right)$ & $70 \times 10^{6}$ \\
\hline$M_{1}$ & Effective mass of fluid (kg) & 0.084 \\
\hline$M_{2}$ & $\begin{array}{l}\text { Effective mass of stiffener with } \\
\text { diaphragm }(\mathrm{kg})\end{array}$ & 0.03 \\
\hline$R_{1}$ & $\begin{array}{l}\text { Helmholtz resonance resistance } \\
\left(\mathrm{kg} \mathrm{s}^{-1} \mathrm{~m}^{-4}\right)\end{array}$ & 100 (assumed) \\
\hline$n$ & Number of stack layers & 2500 \\
\hline$d_{33}$ & $\begin{array}{l}\text { Electro-mechanical charge } \\
\text { (coupling) coefficient }\left(\mathrm{m} \mathrm{V}^{-1}\right)\end{array}$ & $300 \times 10^{-12}[16]$ \\
\hline$\beta_{\mathrm{e}}$ & $\begin{array}{l}\text { Effective bulk modulus of working } \\
\text { fluid (MPa) }\end{array}$ & $0.2,0.3$ \\
\hline$V_{\mathrm{AC}}$ & Amplitude of driving voltage (V) & 120 \\
\hline
\end{tabular}

Equation (22) represents a dynamic system with four poles and one zero that possesses two resonant frequencies, $\omega_{\mathrm{n} 1}$ and $\omega_{\mathrm{n} 2}\left(>\omega_{\mathrm{n} 1}\right)$, that are related to the natural frequencies of the Helmholtz resonator and the pumping chamber with PZT stack transducer, respectively. All parameters and dimensions for simulation are listed in table 1.
Table 1. Simulation parameters and dimensions for simulation of the Helmholtz resonator.

\section{Experimental validation}

Figure 7 represents the overall experimental setup for evaluating the performance of the PHP actuator. The actuator piston is connected to a linear variable displacement transducer (LVDT) to estimate its average velocity, which can be calculated from the measured stroke and stroking time. The sensing signals are analyzed and stored in the dynamic signal analyzer (model HP35665A). In order to compensate for the large capacitive loads of the PZT stack transducer, we customized a two-level driver that switches a large capacitive load between low level (e.g. 0 V) and high level (e.g. 120$150 \mathrm{~V}$ ). With this driving topology, one can provide a quasisinusoidal output voltage with a sufficient current required for the broad bandwidth [8]. The different driving frequencies can be selected through a TTL (transistor-transistor logic) pulse generator.

The frequency response function with the second cylinder diameter $\left(d_{1}=0.05 \mathrm{~m}\right)$ and higher effective bulk modulus (0.3 MPa for Hydrolubric ${ }^{\circledR}$ oil) listed in table 1 is simulated by using equation (22) and compared to the experimental results in figure 8. Although the two-level driver is utilized, the operating frequency is still limited to less than $250 \mathrm{~Hz}$ because of the low current capacity of the two-level driver. From figure 8, there is no doubt that the flow rate has a frequency-dependent characteristic.

To identify the effect of the Helmholtz resonator, two different hydraulic cylinders are assembled to measure the corresponding flow rates. The different Helmholtz cavities (illustrated in figure 9) will cause the variation of the Helmholtz resonance frequency.

As the cavity is changed from type B to type A, the first peak frequency is shifted from the 175 to $240 \mathrm{~Hz}$ with Hydrolubric $^{\circledR}$ oil, as shown in figure 10. Experimental results show good agreement with the simulation results. It can be shown that the volumetric efficiency around $200 \mathrm{~Hz}$ can be significantly improved through the tailoring of the 


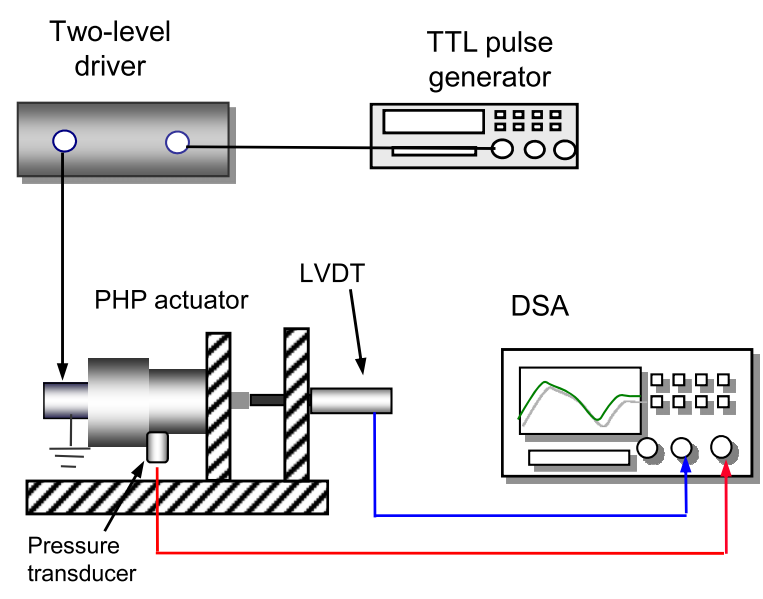

(a) Schematic of configuration

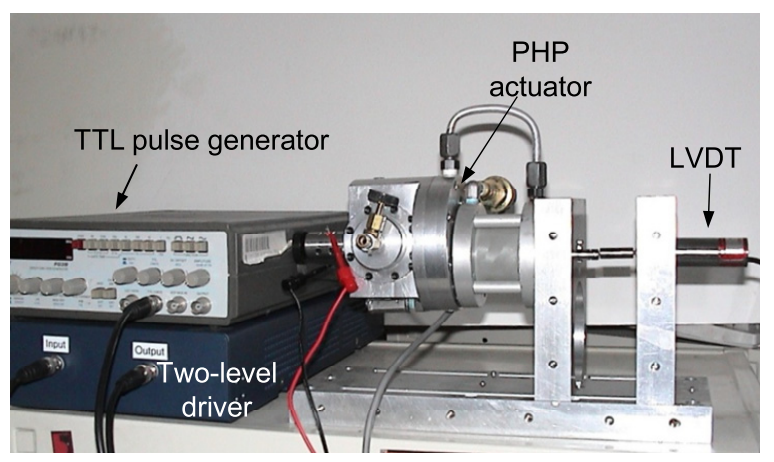

(b) Photograph

Figure 7. Measurement setup for evaluating flow rate performance.

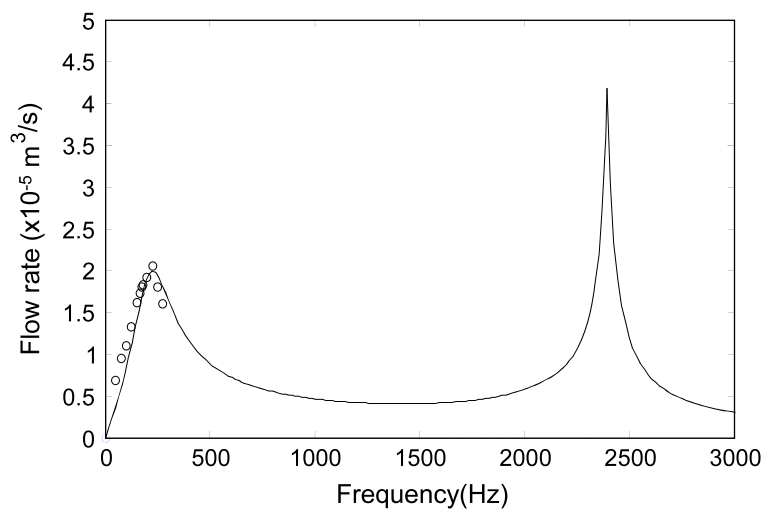

Figure 8. Comparison of flow rate performance $\left(d_{1}=0.05 \mathrm{~m}\right.$; - , simulated; $O$, measured).

Helmholtz resonant frequency. Next, the effect of the effective bulk modulus on Helmholtz resonance is also investigated by replacing the working fluids. Since it is well recognized that the effective bulk modulus of the water-based Hydrolubric ${ }^{\circledR}$ oil is higher than that of petroleum-based hydraulic oil, such as DEXRON-III ${ }^{\circledR}$, under the same pressure $[13,17]$, these two fluids are used alternately as working fluids for the PHP actuator. As in figure 10, the simulated first peak frequency

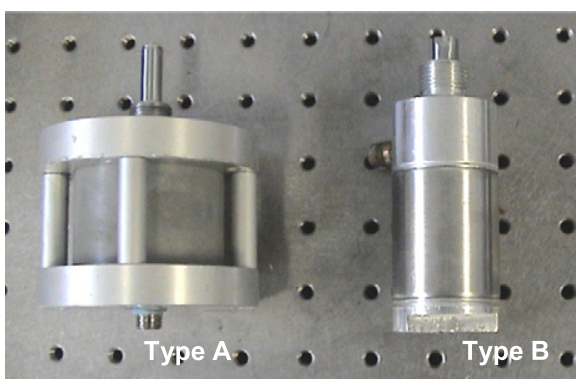

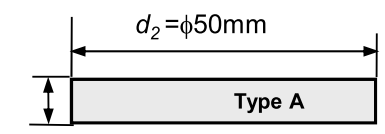

$h_{2}=6 \mathrm{~mm}$

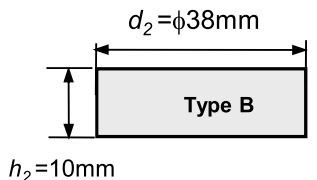

$h_{2}=10 \mathrm{~mm}$
Figure 9. Different Helmholtz cavities of the hydraulic cylinders.

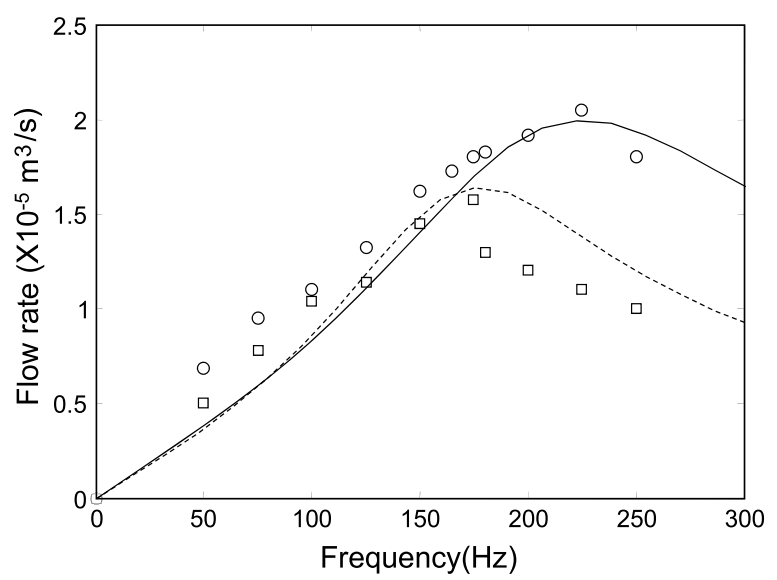

Figure 10. Comparison of flow rate $\left(\right.$ Hydrolubric $^{\circledR}$ oil) with respect to different Helmholtz cavities ( $\square$, type B measured; - - - -, type B simulated; O, type A measured; - , type A simulated).

shown in figure 11 is shifted from 170 to $250 \mathrm{~Hz}$ with the A type cylinder using the working fluid with the higher effective bulk modulus. The experimental results also show good agreement with the simulation predictions.

To further confirm the Helmholtz resonance effect in the PHP actuator, the acoustic sound pressure level (SPL) is experimentally measured by using a microphone. Figure 12 shows the overall SPL measurement stand in an anechoic chamber, in is a shielded room designed to attenuate sound waves. Background noise is not considered in this measurement. Four microphones are located at $0.5 \mathrm{~m}$ from the longitudinal centers of the vertical planes forming the smallest rectangle to measure the average sound pressure level. Air condenser type microphones (Larson Davis laboratories model, sensitivity range of $4-8 \mathrm{mV} \mathrm{Pa}^{-1}$ ) are used in this measurement. The formula for calculating the individual SPL is [14]

$$
\mathrm{SPL}=10 \log _{10}\left[\frac{P_{\mathrm{RMS}}}{P_{\text {ref }}}\right]^{2} \mathrm{~d} B
$$

where $P_{\text {RMS }}$ is the measured RMS sound pressure, $P_{\text {ref }}$ is the reference sound pressure (the commonly used reference sound 


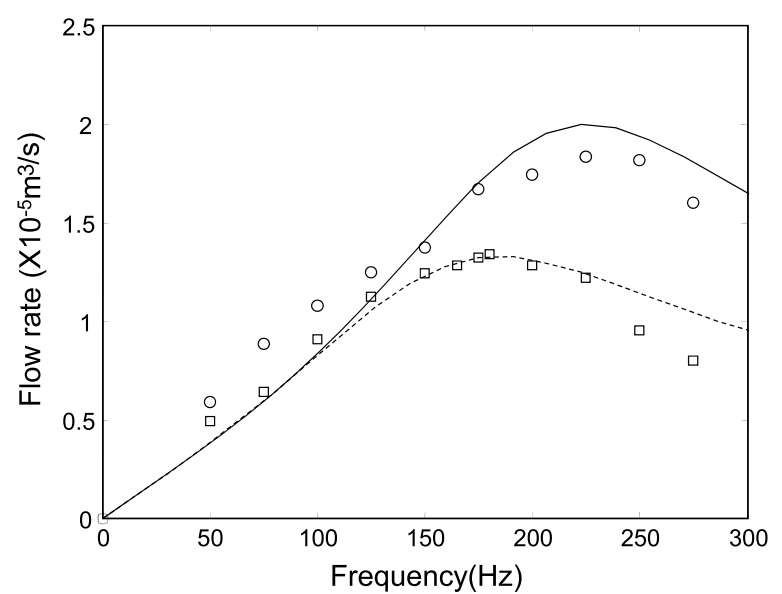

Figure 11. Comparison of flow rate $\left(d_{1}=0.05 \mathrm{~m}\right)$ with respect to different effective bulk moduli ( $\square$, DEXRON-III ${ }^{\circledR}$ measured; - - - -, DEXRON-III ${ }^{\circledR}$ simulated; O, Hydrolubric ${ }^{\circledR}$ measured; - , Hydrolubric ${ }^{\circledR}$ simulated).

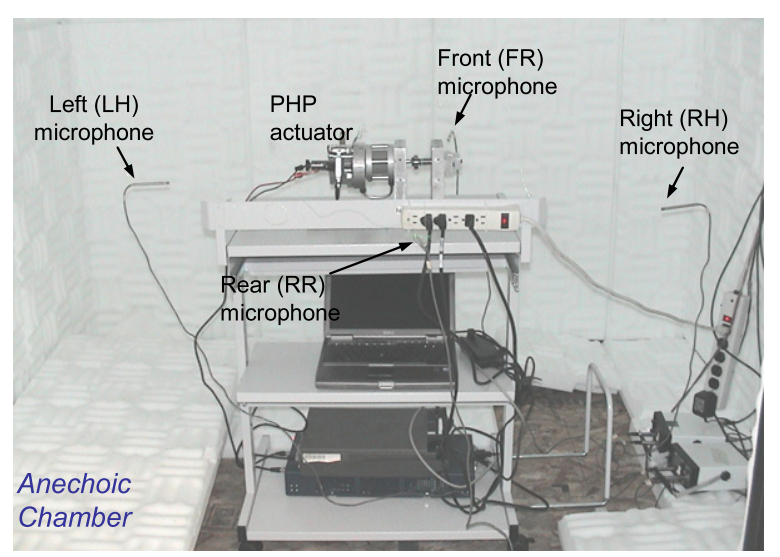

Figure 12. Overall sound pressure level measurement stand located in an anechoic chamber.

pressure in air is $20 \times 10^{-6} \mathrm{~Pa}$ ). Then, the average level of four measurements can be estimated as follows:

$$
\mathrm{SPL}=10 \log _{10}\left[\left(10^{\frac{\mathrm{LH}}{10}}+10^{\frac{\mathrm{RH}}{10}}+10^{\frac{\mathrm{FR}}{10}}+10^{\frac{\mathrm{RR}}{10}}\right) / 4\right] \mathrm{d} B
$$

where $\mathrm{LH}, \mathrm{RH}, \mathrm{FR}$, and RR represent the sound pressure level measured by left, right, front, and rear microphones. The averaged SPL calculated by equation (24) and its power spectral density is illustrated in figure 13. This example corresponds to the case with the type A cylinder and using Hydrolubric $^{\circledR}$ oil. The driving frequency of $200 \mathrm{~Hz}$ is selected to be slightly lower than the expected peak frequency (i.e. 220$230 \mathrm{~Hz}$ ) to prevent an overlap of peaks, and is applied to the PHP actuator. Since the PHP actuator is operated by one-way reed valves with stoppers, there are integer peaked frequencies in the power spectral density spectra (i.e. $200,400 \mathrm{~Hz}$ ), which are related to the contact noises caused by driving the PZT stack transducer. For instance, the peaked frequency of $400 \mathrm{~Hz}$ is generated by the metal contact in the two reed valves. In addition, there is another peak frequency around $220 \mathrm{~Hz}$, which is identical to the Helmholtz resonance frequency shown in
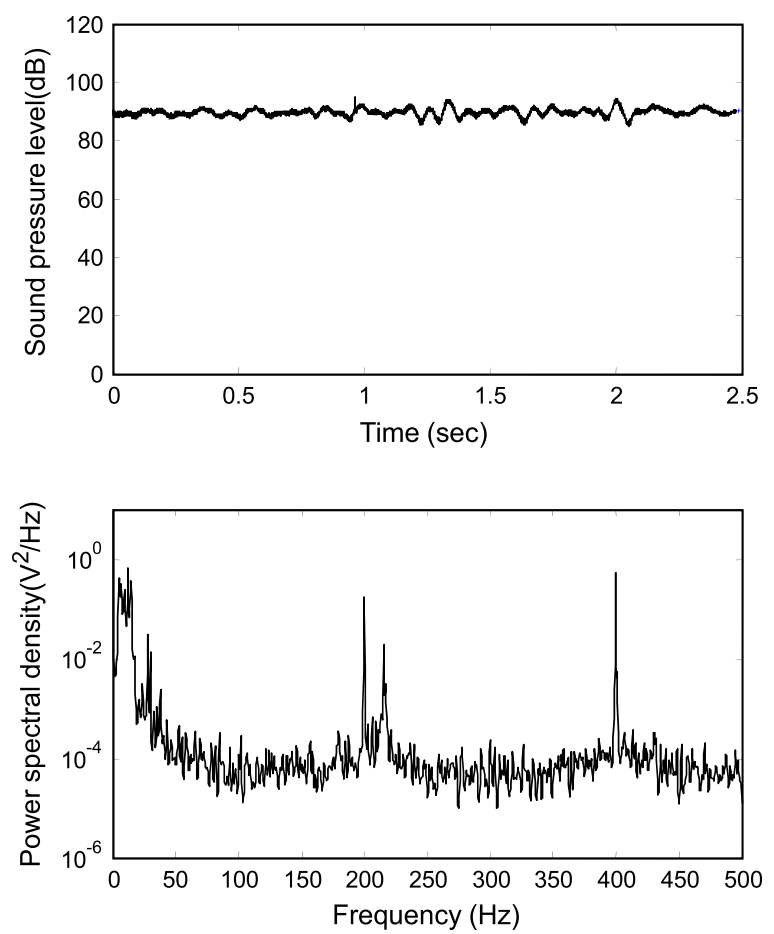

Figure 13. Typical measured sound pressure level and the power spectral density of the PHP actuator.

figure 10. From these results, it can be concluded that the lowest resonance existing in the flow rate of a macro-scale PHP actuator is due to the Helmholtz resonance resulting from the hydraulic circuit configurations using a hydraulic cylinder.

\section{Conclusions}

In this paper, the reason for the degradation of volumetric efficiency in PHP actuators is investigated and methods to improve the PHP performance are presented. It is clear that the large cavity in the double-acting cylinder acts as a Helmholtz resonator when the PHP is combined with hydraulic circuitswhich normally consist of a hydraulic cylinder. Based on the analysis and experimental results, it is concluded that simple modifications of the device design or working fluids will allow us to improve the volumetric efficiency of the PHP and enhance the actuator performance by extending its effective frequency bandwidth.

\section{Acknowledgment}

This work is supported by the Ford Motor Company University Research Program.

\section{References}

[1] Konishi K, Yoshimura T, Hashimoto K and Yamamoto N 1993 Hydraulic actuators driven by piezoelectric elements (1st report, trial piezoelectric pump and its maximum power) J. Japan. Soc. Mech. Eng. C 59 213-20

[2] Mauck L D and Lynch C S 2000 Piezoelectric hydraulic pump development J. Intell. Mater. Syst. Struct. 11 758-64 
[3] Nasser J, Leo D J and Cundey H 2000 Compact piezohydraulic actuation system Smart Structures and Materials 2000; Proc. SPIE 3991 312-22

[4] Oates W S and Lynch C S 2001 Piezoelectric hydraulic pump system dynamic model J. Intell. Mater. Syst. Struct. $12737-44$

[5] Sirohi J and Chopra I 2003 Design and development of a high pumping frequency piezoelectric-hydraulic hybrid actuator J. Intell. Mater. Syst. Struct. 14 135-47

[6] Lee D G, Or S W and Carman G P 2004 Design of a piezoelectric-hydraulic pump with active valves J. Intell. Mater. Syst. Struct. 15 107-15

[7] Tan H, Hurst W and Leo D J 2005 Performance modeling of a piezohydraulic actuation system with active valves J. Smart Mater. Struct. 14 91-110

[8] Kim G W and Wang K W 2009 Switching sliding mode force tracking control of piezoelectric-hydraulic pump based friction element actuation systems for automotive transmissions Smart Mater. Struct. 18085004

[9] Kan J, Tang K, Ren Y, Zhu G and Li P 2009 Study on a piezohydraulic pump for linear actuators Sensors Actuators A 149 331-9
[10] Wereley N M and Chaudhuri A 2010 Experimental validation of a hybrid electrostrictive hydraulic actuator analysis $A S M E$ J. Vib. Acoust. 132021006

[11] Griffin S, Lane S A and Steve H 2001 Coupled Helmholtz resonators for acoustic attenuation ASME J. Vib. Acoust. $12311-7$

[12] Dupere I D J and Dowling A P 2005 The use of Helmholtz resonators in a practical combustor ASME J. Eng. Gas Turbines Power 127 268-75

[13] Kim G W and Wang K-W 2009 On-line estimation of effective bulk modulus in fluid power systems using piezoelectric transducer impedance J. Intell. Mater. Syst. Struct. 20 2101-6

[14] Kinsler L E, Frey A R, Coppeans A B and Sanders J V 2000 Fundamentals of Acoustics 4th edn (New York: Wiley)

[15] Manring N D 2005 Hydraulic Control Systems (New York: Wiley)

[16] APC International, Ltd 2006 Product Catalog http://www. americanpiezo.com

[17] Houghton International Inc 2008 Hydrolubric ${ }^{\circledR}$ high water content fluids (HWCF) brochure http://www.houghtonintl. com 